Copyright (C) 2015 IEEE. Personal use of this material is permitted. Permission from IEEE must be obtained for all other uses, in any current or future media, including reprinting/republishing this material for advertising or promotional purposes, creating new collective works, for resale or redistribution to servers or lists, or reuse of any copyrighted component of this work in other works. 


\title{
Fairness Considerations in Full-Duplex MIMO Interference Channels
}

\author{
Ali Cagatay Cirik*, Yue Rong ${ }^{\dagger}$, Yingbo Hua ${ }^{\ddagger}$, and Matti Latva-aho* \\ * Centre for Wireless Communications, University of Oulu, Oulu, Finland \\ $\dagger$ Department of Electrical and Computer Engineering, Curtin University, Bentley, WA 6102, Australia \\ $\ddagger$ Department of Electrical Engineering, University of California Riverside, Riverside, CA, 92521 \\ Email: acirik@ee.oulu.fi,y.rong@curtin.edu.au,yhua@ee.ucr.edu, matti.latva-aho@ee.oulu.fi
}

\begin{abstract}
In this paper, we address the proportional fair (PF) issue of a $K$ link full-duplex (FD) multiple-input multiple-output (MIMO) interference channel, where each link consists of two FD nodes exchanging information simultaneously. The nodes in each pair suffer from self-interference due to operating in FD mode, and inter-user interference from the nodes in other links due to simultaneous transmission from each link. The PF issue is important for networks with asymmetric topology and/or asymmetric traffic demands. We demonstrate that the proposed algorithm provides a good trade-off between sum achievable rate and rate distribution for asymmetric links, and moreover we show that the sum-rate achieved by FD mode is higher than the sum-rate achieved by baseline half-duplex (HD) schemes.
\end{abstract}

Index Terms-Full-duplex, MIMO interference channel, proportional fairness, self interference.

\section{INTRODUCTION}

Recently, full-duplex (FD) communication, which enables receiving and transmitting on the same channel simultaneously, has been proposed to meet the spectral efficiency requirements of next generation wireless communication systems. Spectral efficiency gain of FD systems over half-duplex (HD) systems, in which reception and transmission is performed on orthogonal channels, has been studied theoretically in [1]-[8], and experimentally in [9]-[14].

The limiting factor on the performance of FD systems is the self-interference at the front-end of the receiver created by the signal leakage from the transmitter antennas of a FD node to its own receiver antennas. Promising results from experimental research that demonstrate the feasibility of FD radios using the off-the-shelf hardware are available in [9]-[14]. Since the self-interference cannot be canceled completely in practice due to channel estimation errors, and imperfections of radio devices such as amplifier non-linearity, phase noise, and I/Q channel imbalance, optimization problems (power allocation, transceiver beamforming, etc.) related to FD systems under this residual self-interference were considered in [1]-[8].

To the best of our knowledge, all the papers on FD bidirectional systems consider a single pair of FD nodes, exchanging information simultaneously [1]-[8], and no paper has studied FD systems under multiple pair of nodes, i.e.

The research leading to these results has received funding in part from the European Union Seventh Framework Programme (FP7/2007-2013) under grant agreement number 316369 - project DUPLO, and in part from the Academy of Finland under Grant 260755 - Project Juliet.
FD MIMO interference channel. Recently, the interest on MIMO channels has migrated from the point-to-point MIMO and MIMO downlink channel, to the MIMO interference channel, since it is the inherent model behind many practical problems [15], and the performance of cellular communication systems where each cell causes interference to other cells can be studied by focusing on MIMO interference channels [16].

In this paper, we consider a $K$ link MIMO interference channel, where each link has two FD nodes exchanging information simultaneously. The nodes in each pair suffer from self-interference due to operating in FD mode, and inter-user interference due to simultaneous transmission from each link. In practice, due to the different distances (and corresponding path losses) of the nodes, fairness issue among the nodes may arise when allocating transmission resources to nodes to maximize the sum-rate, and thus there is generally a trade-off between system throughput and fairness. Therefore, the fairness issue is particularly important for networks with asymmetric topology and/or asymmetric traffic demands.

To address the fairness issue for FD MIMO interference channels, we use a proportionally fair (PF) utility function, which was proposed in [17] and later also was applied in [18][20]. We maximize the sum of the logarithm of the achievable rate of each node in the network subject to individual power constraints at each node. The PF notion is automatically embedded in such a utility function, since maximizing the logarithmic utility function, yields a good balance between system throughput and fairness [20]. We develop a Gradient Projection (GP) method [21] to solve this non-convex optimization problem, and demonstrate that incorporating the PF notion into the sum-rate maximization problem typically yields a near maximum sum rate for asymmetric networks while a relatively even distribution of link rates can be maintained. Moreover, simulation results show that the proposed FD system outperforms the baseline HD systems.

The following notations are used in this paper. Matrices and vectors are denoted as bold capital and lowercase letters, respectively. $(\cdot)^{T}$ and $(\cdot)^{H}$ are the transpose and the conjugate transpose, respectively. $\mathbf{I}_{N}$ is the $N$ by $N$ identity matrix; $\operatorname{tr}(\cdot)$ is the trace; $|\cdot|$ is the determinant; $\operatorname{diag}(\mathbf{A})$ is the diagonal matrix with the same diagonal elements as $\mathbf{A} . \mathcal{C N}\left(\mu, \sigma^{2}\right)$ denotes a complex Gaussian distribution with mean $\mu$ and variance $\sigma^{2} . \perp$ denotes the statistical independence. 


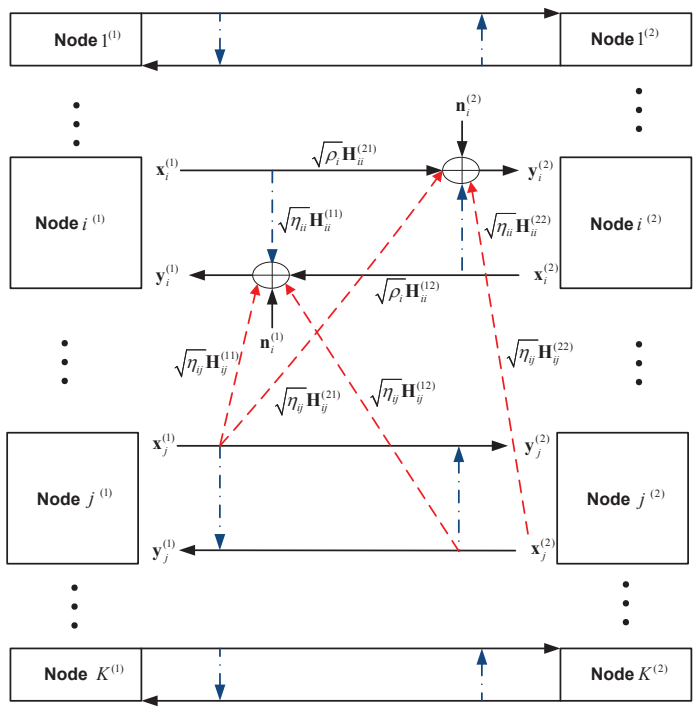

Fig. 1. Bi-directional full-duplex MIMO interference channel.

\section{System Model}

In this section, we describe the system model of a FD MIMO interference channel as seen in Fig. 1. Each pair in the system is equipped with multiple antennas and exchanges information simultaneously in a two way communication. We assume that each node has $N$ physical antennas that can be used for simultaneous receiving and transmitting at the same carrier frequency [14].

As illustrated in Fig. 1, the node $i^{(a)}, i \in\{1, \ldots, K\}$, $a \in\{1,2\}$ receives signals from all the transmitters in the system via MIMO channels. $\mathbf{H}_{i i}^{(a b)} \in \mathbb{C}^{N \times N}$ is the desired channel between node $a$ and $b$ of the $i$-th transmitter-receiver pair, where $(a, b) \in\{1,2\}$ and $b \neq a$. $\mathbf{H}_{i i}^{(a a)} \in \mathbb{C}^{N \times N}, a \in$ $\{1,2\}$ denotes the self-interference channel of the node $i^{(a)}$. $\mathbf{H}_{i j}^{(a b)} \in \mathbb{C}^{N \times N},(a, b) \in\{1,2\}$ denotes the interference channel from the transmitter antennas of the node $b$ in the $j$-th pair to the receiver antennas of the node $a$ in the $i$-th pair, $(i, j) \in\{1, \ldots, K\}$ and $j \neq i$. All the channel matrices are assumed to be mutually independent and the entries of each matrix are independent and identically distributed (i.i.d.) circular complex Gaussian variables with zero mean and unit variance.

We consider a FD bi-directional MIMO interference channel that suffers from self-interference and interference from other pairs. Thus, node $i^{(a)}$ receives a combination of the signals transmitted by all the transmitters and noise. The $N \times 1$ received signal at node $i^{(a)}$ is written as

$$
\begin{aligned}
\mathbf{y}_{i}^{(a)}= & \sqrt{\rho}_{i} \mathbf{H}_{i i}^{(a b)}\left(\mathbf{x}_{i}^{(b)}+\mathbf{c}_{i}^{(b)}\right)+\sqrt{\eta}_{i i} \mathbf{H}_{i i}^{(a a)}\left(\mathbf{x}_{i}^{(a)}+\mathbf{c}_{i}^{(a)}\right) \\
& +\sum_{j \neq i}^{K} \sum_{c=1}^{2} \sqrt{\eta_{i j}} \mathbf{H}_{i j}^{(a c)}\left(\mathbf{x}_{j}^{(c)}+\mathbf{c}_{j}^{(c)}\right)+\mathbf{e}_{i}^{(a)} \\
& +\mathbf{n}_{i}^{(a)}, i \in\{1, \ldots, K\}, \quad(a, b) \in\{1,2\}, a \neq b, \quad(1)
\end{aligned}
$$

where $\mathbf{x}_{i}^{(a)} \sim \mathcal{C N}\left(\mathbf{0}, \mathbf{Q}_{i}^{(a)}\right)$ is the signal vector transmitted by node $i^{(a)}, i \in\{1, \ldots, K\}, a \in\{1,2\}$, and $\mathbf{n}_{i}^{(a)} \in \mathbb{C}^{N}$ is the additive white Gaussian noise (AWGN) vector at node $i^{(a)}$ with zero mean and unit covariance matrix. In (1), $\rho_{i}$ denotes the average power gain of the $i$-th transmitter-receiver pair, $\eta_{i i}$ denotes the average power gain of the self-interference channel at the $i$-th pair, and $\eta_{i j}$ denotes the average power gain of the interference channel between the nodes at the $i$-th and $j$-th pair.

In (1), $\mathbf{c}_{i}^{(a)} \in \mathbb{C}^{N}, i \in\{1, \ldots, K\}, a \in\{1,2\}$ is the transmitter noise at the transmitter antenna of node $i^{(a)}$, which models the effect of limited transmitter limited dynamic range (DR) and closely approximates the effects of additive poweramplifier noise, non-linearities in the DAC and phase noise. The covariance matrix of $\mathbf{c}_{i}^{(a)}$ is given by $\kappa(\kappa \ll 1)$ times the energy of the intended signal at each transmit antenna [1]. In particular $\mathbf{c}_{i}^{(a)}$ can be modeled as

$$
\mathbf{c}_{i}^{(a)} \sim \mathcal{C N}\left(\mathbf{0}, \kappa \operatorname{diag}\left(\mathbf{Q}_{i}^{(a)}\right)\right), \quad \mathbf{c}_{i}^{(a)} \perp \mathbf{x}_{i}^{(a)} .
$$

In (1), $\mathbf{e}_{i}^{(a)} \in \mathbb{C}^{N}, i \in\{1, \ldots, K\}, a \in\{1,2\}$ is the additive receiver distortion at the receiver antennas of node $i^{(a)}$, which models the effect of limited receiver DR and closely approximates the combined effects of additive gaincontrol noise, non-linearities in the ADC and phase noise. The covariance matrix of $\mathbf{e}_{i}^{(a)}$ is given by $\beta(\beta \ll 1)$ times the energy of the undistorted received signal at each receive antenna [1]. In particular, $\mathbf{e}_{i}^{(a)}$ can be modeled as

$$
\mathbf{e}_{i}^{(a)} \sim \mathcal{C N}\left(\mathbf{0}, \beta \operatorname{diag}\left(\boldsymbol{\Phi}_{i}^{(a)}\right)\right), \quad \mathbf{e}_{i}^{(a)} \perp \mathbf{u}_{i}^{(a)},
$$

where $\boldsymbol{\Phi}_{i}^{(a)}=\operatorname{Cov}\left\{\mathbf{u}_{i}^{(a)}\right\}$ and $\mathbf{u}_{i}^{(a)}$ is the undistorted received vector at the node $i^{(a)}$, i.e. $\mathbf{u}_{i}^{(a)}=\mathbf{y}_{i}^{(a)}-\mathbf{e}_{i}^{(a)}$. This transmitter/receiver distortion model is valid, since it was shown by hardware measurements in [22] and [23] that the non-ideality of the transmitter and receiver chain can be approximated by an independent Gaussian noise model, respectively.

Since node $i^{(a)}$ knows the interfering signal $\mathbf{x}_{i}^{(a)}$ and channel matrix $\mathbf{H}_{i i}^{(a a)}$, the self-interference term $\sqrt{\eta}_{i i} \mathbf{H}_{i i}^{(a a)} \mathbf{x}_{i}^{(a)}$ can be cancelled [1]. The interference canceled signal can then be written as

$$
\begin{aligned}
\tilde{\mathbf{y}}_{i}^{(a)} & =\mathbf{y}_{i}^{(a)}-\sqrt{\eta}_{i i} \mathbf{H}_{i i}^{(a a)} \mathbf{x}_{i}^{(a)} \\
& =\sqrt{\rho}_{i} \mathbf{H}_{i i}^{(a b)} \mathbf{x}_{i}^{(b)}+\mathbf{v}_{i}^{(a)}
\end{aligned}
$$

where $\mathbf{v}_{i}^{(a)}$ is the unknown interference components of (4) after self-interference cancellation and is given by

$$
\begin{aligned}
\mathbf{v}_{i}^{(a)}= & \sqrt{\rho}_{i} \mathbf{H}_{i i}^{(a b)} \mathbf{c}_{i}^{(b)}+\sqrt{\eta}_{i i} \mathbf{H}_{i i}^{(a a)} \mathbf{c}_{i}^{(a)}+\mathbf{e}_{i}^{(a)}+\mathbf{n}_{i}^{(a)} \\
& +\sum_{j \neq i}^{K} \sum_{c=1}^{2} \sqrt{\eta_{i j}} \mathbf{H}_{i j}^{(a c)}\left(\mathbf{x}_{j}^{(c)}+\mathbf{c}_{j}^{(c)}\right) .
\end{aligned}
$$

Similar to the derivation in [1], using (2)-(3), $\tilde{\Sigma}_{i}^{(a)}$, the covariance matrix of $\mathbf{v}_{i}^{(a)}$, can be approximated, under $\kappa \ll 1$ and $\beta \ll 1$, as in (6) shown at the bottom of the following page. 
The noise $\mathbf{v}_{i}^{(a)}$ is non-Gaussian because of the transmitter/receiver distortion in (5). However, it is known that the Gaussian distribution is the worst case from a mutualinformation perspective among all noise distributions [24], and thus assuming $\mathbf{v}_{i}^{(a)}$ as Gaussian, gives us the lower bound of mutual information [24]. The lower bound of the achievable rate of the node $i^{(a)}$, under Gaussian signaling, can be written as

$$
I_{i}^{(a)}=\log _{2}\left|\mathbf{I}_{N}+\rho_{i} \mathbf{H}_{i i}^{(a b)} \mathbf{Q}_{i}^{(b)}\left(\mathbf{H}_{i i}^{(a b)}\right)^{H}\left(\tilde{\boldsymbol{\Sigma}}_{i}^{(a)}\right)^{-1}\right| .
$$

\section{Proportional FAir Based Scheme}

Following the idea used in [18]-[20], PF based optimization problem can be formulated as:

$$
\begin{aligned}
\max _{\overline{\mathbf{Q}}} & \underbrace{\sum_{i=1}^{K} \sum_{a=1}^{2} \ln \left(I_{i}^{(a)}\right)}_{I(\overline{\mathbf{Q}})} \\
\text { s.t } & \operatorname{tr}\left\{\mathbf{Q}_{i}^{(b)}\right\} \leq P_{i}^{(b)}, i=1, \ldots, K, b=1,2, \\
& \mathbf{Q}_{i}^{(b)} \geq 0, i=1, \ldots, K, b=1,2,
\end{aligned}
$$

where we use the following definition for a stacked matrix $\overline{\mathbf{Q}}$ : $\overline{\mathbf{Q}} \triangleq\left[\left(\mathbf{Q}_{1}^{(1)}\right)^{T},\left(\mathbf{Q}_{1}^{(2)}\right)^{T}, \ldots,\left(\mathbf{Q}_{K}^{(1)}\right)^{T},\left(\mathbf{Q}_{K}^{(2)}\right)^{T}\right]^{T}, P_{i}^{(b)}$ in (9) is the transmit power constraint at the node $i^{(b)}$.

We now develop an algorithm to find a local optimal solution to the problem (8)-(10) by following the GP technique [21]. Two major parts of the GP algorithm are: the computation of the gradient of the objective function, and the projection of the gradient onto a feasible set. The gradient of the objective function with respect to $\mathbf{Q}_{i}^{(b)}$ is written as

$$
\mathbf{G}_{i}^{(b)}=2\left[\frac{\partial I(\overline{\mathbf{Q}})}{\partial \mathbf{Q}_{i}^{(b)}}\right]^{*}
$$

where the partial derivative $\frac{\partial I(\overline{\mathbf{Q}})}{\partial \mathbf{Q}_{i}^{(b)}}$ is shown in (12) at the bottom of the following page. In (12), $\mathbf{X}_{i}^{(a)}=$ $\rho_{i} \mathbf{H}_{i i}^{(a b)} \mathbf{Q}_{i}^{(b)}\left(\mathbf{H}_{i i}^{(a b)}\right)^{H},(a, b) \in(1,2), a \neq b$, and $\boldsymbol{\Pi}_{i}^{(a)}$, socalled interference sensitivity matrix, is defined in (13) at the bottom of the following page.

After the gradient $\mathbf{G}_{i}^{(b)}$ is obtained, the covariance matrix $\mathbf{Q}_{i}^{(b)}$ is updated to $\hat{\mathbf{Q}}_{i}^{(b)}$ by $\hat{\mathbf{Q}}_{i}^{(b)}=\mathbf{Q}_{i}^{(b)}+s \mathbf{G}_{i}^{(b)}$ where $s$

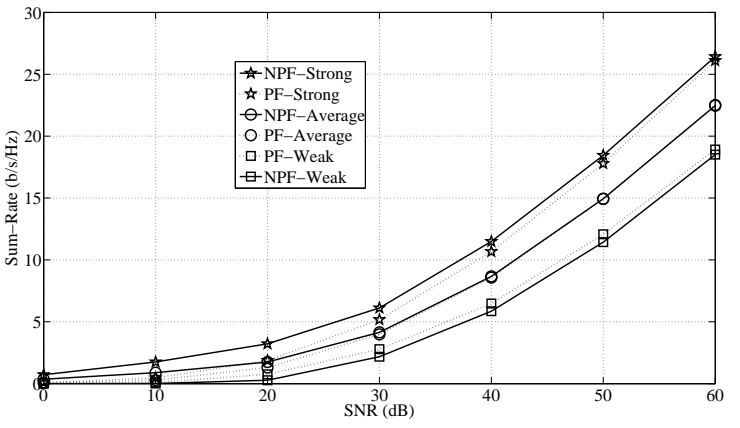

Fig. 2. Average per-user rate comparison of PF and NPF schemes. INR = $20 \mathrm{~dB}, \mathrm{INR}_{\mathrm{SI}}=40 \mathrm{~dB}, N=3, K=10, \kappa=\beta=-40 \mathrm{~dB}$.

is a scalar. Now we need to project the matrix $\hat{\mathbf{Q}}_{i}^{(b)}$ onto the feasible region defined by (9) and (10). Projecting $\hat{\mathbf{Q}}_{i}^{(b)}$ onto the feasible region, and a general form of the GP algorithm (steps for the update of power allocation, Armijo rule, convergence criterion, etc.) is similar to that in [21], which we will not repeat.

\section{NumERICAL RESUlTS}

We compare our proposed algorithm with the algorithm that directly maximizes the sum-rate (the same optimization problem in (8)-(10) without the logarithm term, ln in (8)), named as Non-Proportional Fairness (NPF). We assume that the nodes in the system have the same transmit power constraint, i.e., $P_{i}^{(b)}=N, i=1, \ldots, K, b=1,2$. We define signal-to-noise ratio (SNR) of the nodes in the $i$-th pair as $\mathrm{SNR}_{i} \triangleq \rho_{i} N$, and the nominal interference-to-noise ratio (INR) from the nodes in the $j$-th pair to the nodes in the $i$-th pair as $\mathrm{INR}_{i j}=\eta_{i j} N, i \neq j$. We consider an asymmetric network with $K / 2$ strong links and $K / 2$ weak links, i.e.,

$$
\mathrm{SNR}_{i}= \begin{cases}\mathrm{SNR}+10 \mathrm{~dB} & i=1, \ldots, \frac{K}{2} \\ \mathrm{SNR} & i=\frac{K}{2}+1, \ldots, K\end{cases}
$$

We also set $\mathrm{INR}_{i j}=\mathrm{INR}, i, j=1, \ldots, K, i \neq j$, and $\mathrm{INR}_{i i}$ which corresponds the INR of the self-interference channel at the nodes in the $i$-th pair, is denoted as $\mathrm{INR}_{\mathrm{SI}}$. The performance of all algorithms are compared in terms of (a) the per link rate averaged over strong links, (b) the per link rate averaged over weak links, and (c) the per link rate

$$
\begin{aligned}
\tilde{\mathbf{\Sigma}}_{i}^{(a)} \approx & \rho_{i} \kappa \mathbf{H}_{i i}^{(a b)} \operatorname{diag}\left(\mathbf{Q}_{i}^{(b)}\right)\left(\mathbf{H}_{i i}^{(a b)}\right)^{H}+\eta_{i i} \kappa \mathbf{H}_{i i}^{(a a)} \operatorname{diag}\left(\mathbf{Q}_{i}^{(a)}\right)\left(\mathbf{H}_{i i}^{(a a)}\right)^{H}+\beta \rho_{i} \operatorname{diag}\left(\mathbf{H}_{i i}^{(a b)} \mathbf{Q}_{i}^{(b)}\left(\mathbf{H}_{i i}^{(a b)}\right)^{H}\right) \\
& +\beta \eta_{i i} \operatorname{diag}\left(\mathbf{H}_{i i}^{(a a)} \mathbf{Q}_{i}^{(a)}\left(\mathbf{H}_{i i}^{(a a)}\right)^{H}\right)+\sum_{j \neq i}^{K} \sum_{c=1}^{2} \beta \eta_{i j} \operatorname{diag}\left(\mathbf{H}_{i j}^{(a c)} \mathbf{Q}_{j}^{(c)}\left(\mathbf{H}_{i j}^{(a c)}\right)^{H}\right) \\
& +\sum_{j \neq i}^{K} \sum_{c=1}^{2} \eta_{i j} \mathbf{H}_{i j}^{(a c)}\left(\mathbf{Q}_{j}^{(c)}+\kappa \operatorname{diag}\left(\mathbf{Q}_{j}^{(c)}\right)\right)\left(\mathbf{H}_{i j}^{(a c)}\right)^{H}+\mathbf{I}_{N}
\end{aligned}
$$


averaged over all links, defined as follows:

$$
\begin{aligned}
C_{\text {strong }} & \triangleq \frac{1}{L K} \sum_{l=1}^{L} \sum_{i=1}^{\frac{K}{2}} \sum_{a=1}^{2} I_{i}^{(a)(l)}, \\
C_{\text {weak }} & \triangleq \frac{1}{L K} \sum_{l=1}^{L} \sum_{i=\frac{K}{2}+1}^{K} \sum_{a=1}^{2} I_{i}^{(a)(l)}, \\
C_{\text {average }} & \triangleq \frac{1}{2 L K} \sum_{l=1}^{L} \sum_{i=1}^{K} \sum_{a=1}^{2} I_{i}^{(a)(l)},
\end{aligned}
$$

where $I_{i}^{(a)(l)}$ denotes the achievable rate of the node $i^{(a)}$ in (7) for the $l$-th realization. The parameters for the GP algorithm is $s=1, \sigma=0.1, \theta=0.5$, and $\epsilon=0.01$.

Fig. 2 illustrates the per-link achievable rate for PF and NPF schemes with respect to SNR over $L=100$ realizations. Since PF algorithm is not designed to maximize the sum-rate, it yields lower $C_{\text {average }}$ than NPF, however the difference is very small. It can be seen that the difference $C_{\text {strong }}-C_{\text {weak }}$ is big in NPF scheme compared to PF scheme, and thus PF scheme avoids starvation of users at low SNR. Therefore it can be concluded that PF scheme appears to have the best trade-off between sum-rate and fairness.

In our last example, we have compared our system, in which all the pairs operate in FD mode, and transmit at the same time (both self-interference, and inter-user interference exist) with the following baseline systems:

- FD-TDMA: All the pairs in the system operate in FD mode, but transmit on different time slots, like TDMA (only self-interference, no inter-user interference).

- HD: All the pairs in the system operate in HD mode, but transmit at the same time (only inter-user interference, no

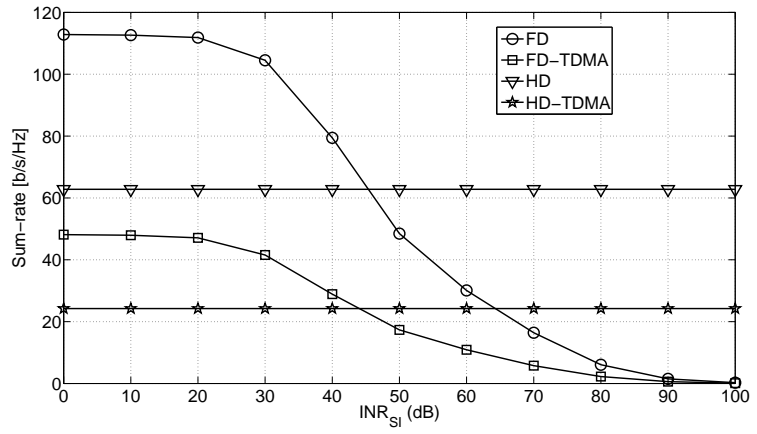

Fig. 3. Sum-rate comparison for different schemes versus $\mathrm{INR}_{\mathrm{SI}}$. Here $K=4, N=4, \mathrm{SNR}=25 \mathrm{~dB}, \mathrm{INR}=0 \mathrm{~dB}, \kappa=\beta=-30 \mathrm{~dB}$.

self-interference). Particularly, in the first (second) time slot, all the nodes on the left (right) in Fig. 1 transmit to their pairs on the right (left).

- HD-TDMA: All the nodes transmit sequentially, so we need $2 K$ time slots (no inter-user interference, no selfinterference).

The effect of the self-interference on the proposed and baseline schemes is examined in Fig. 3. The performance of FD system drops below that of HD and HD-TDMA schemes around $\mathrm{INR}_{\mathrm{SI}}=45 \mathrm{~dB}$ and $\mathrm{INR}_{\mathrm{SI}}=65 \mathrm{~dB}$, respectively.

\section{CONCLUSION}

In this paper, we have investigated the fairness issue of FD MIMO interference channels for asymmetric networks. The proposed algorithm is shown to provide a trade-off between sum-rate and fair distribution of data rates in comparison to the NPF algorithm that maximizes the sum-rate directly.

$$
\begin{aligned}
& \frac{\partial I(\overline{\mathbf{Q}})}{\partial \mathbf{Q}_{i}^{(b)}} \\
= & \frac{1}{I_{i}^{(a)}}\left[\frac{1}{\ln 2} \rho_{i}\left(\mathbf{H}_{i i}^{(a b)}\right)^{H}\left(\tilde{\boldsymbol{\Sigma}}_{i}^{(a)}+\mathbf{X}_{i}^{(a)}\right)^{-1} \mathbf{H}_{i i}^{(a b)}-\rho_{i} \kappa \operatorname{diag}\left(\left(\mathbf{H}_{i i}^{(a b)}\right)^{H} \mathbf{\Pi}_{i}^{(a)} \mathbf{H}_{i i}^{(a b)}\right)-\rho_{i} \beta\left(\mathbf{H}_{i i}^{(a b)}\right)^{H} \operatorname{diag}\left(\mathbf{\Pi}_{i}^{(a)}\right) \mathbf{H}_{i i}^{(a b)}\right]^{T} \\
& -\sum_{j \neq i}^{K} \sum_{c=1}^{2} \frac{1}{I_{j}^{(c)}}\left[\eta_{j i}\left(\mathbf{H}_{j i}^{(c b)}\right)^{H} \boldsymbol{\Pi}_{j}^{(c)} \mathbf{H}_{j i}^{(c b)}+\eta_{j i} \kappa \operatorname{diag}\left(\left(\mathbf{H}_{j i}^{(c b)}\right)^{H} \boldsymbol{\Pi}_{j}^{(c)} \mathbf{H}_{j i}^{(c b)}\right)+\eta_{j i} \beta\left(\mathbf{H}_{j i}^{(c b)}\right)^{H} \operatorname{diag}\left(\mathbf{\Pi}_{j}^{(c)}\right) \mathbf{H}_{j i}^{(c b)}\right]^{T} \\
& -\frac{1}{I_{i}^{(b)}}\left[\eta_{i i} \kappa \operatorname{diag}\left(\left(\mathbf{H}_{i i}^{(b b)}\right)^{H} \mathbf{\Pi}_{i}^{(b)} \mathbf{H}_{i i}^{(b b)}\right)+\eta_{i i} \beta\left(\mathbf{H}_{i i}^{(b b)}\right)^{H} \operatorname{diag}\left(\mathbf{\Pi}_{i}^{(b)}\right) \mathbf{H}_{i i}^{(b b)}\right]^{T}
\end{aligned}
$$

$$
\begin{aligned}
\boldsymbol{\Pi}_{i}^{(a)} & =-\left(\frac{\partial}{\partial \boldsymbol{\Sigma}_{i}^{(a)}} \log _{2}\left|\mathbf{I}_{N}+\rho_{i} \mathbf{H}_{i i}^{(a b)} \mathbf{Q}_{i}^{(b)}\left(\mathbf{H}_{i i}^{(a b)}\right)^{H}\left(\tilde{\boldsymbol{\Sigma}}_{i}^{(a)}\right)^{-1}\right|\right)^{T} \\
& =\log _{2} e\left(\frac{1}{\partial \boldsymbol{\Sigma}_{i}^{(a)}} \operatorname{tr}\left\{\left(\mathbf{I}_{N}+\mathbf{X}_{i}^{(a)}\left(\tilde{\boldsymbol{\Sigma}}_{i}^{(a)}\right)^{-1}\right)^{-1} \mathbf{X}_{i}^{(a)}\left(\tilde{\boldsymbol{\Sigma}}_{i}^{(a)}\right)^{-1} \partial \tilde{\boldsymbol{\Sigma}}_{i}^{(a)}\left(\tilde{\boldsymbol{\Sigma}}_{i}^{(a)}\right)^{-1}\right\}\right)^{T} \\
& =\log _{2} e\left(\left(\tilde{\boldsymbol{\Sigma}}_{i}^{(a)}\right)^{-1}-\left(\mathbf{X}_{i}^{(a)}+\tilde{\boldsymbol{\Sigma}}_{i}^{(a)}\right)^{-1}\right) .
\end{aligned}
$$




\section{REFERENCES}

[1] B. P. Day, A. R. Margetts, D. W. Bliss, and P. Schniter, "Full-duplex bidirectional MIMO: Achievable rates under limited dynamic range," IEEE Trans. Signal Process., vol. 60, no. 7, pp. 3702-3713, July 2012.

[2] A. C. Cirik, Y. Rong, and Y. Hua, "Achievable rates of full-duplex MIMO radios in fast fading channels with imperfect channel estimation", IEEE Trans. Signal Process., vol. 62, pp. 3874-3886, Aug. 2014.

[3] A. C. Cirik, R. Wang, Y. Hua, and M. Latva-aho, "Weighted sumrate maximization for full-duplex MIMO interference channels," accepted IEEE Trans. Commun., Jan. 2015.

[4] A. C. Cirik, R. Wang, Y. Rong, and Y. Hua, "MSE based transceiver designs for bi-directional full-duplex MIMO systems," IEEE Workshop on Signal Process. Advances in Wireless Commun. (SPAWC'2014), pp. 384388, June, 2014.

[5] S. Huberman, and T. Le-Ngoc, "Sequential convex programming for fullduplex single-user MIMO systems," IEEE Int. Conf. Commun. (ICC), pp. 5078-5082, June 2014.

[6] W. Li, J. Lilleberg, and K. Rikkinen, "On rate region analysis of half- and full-duplex OFDM communication links," IEEE J. Sel. Areas Commun., vol. 32, no. 9, pp. 1688-1698, Sept. 2014.

[7] J. Zhang, O. Taghizadeh, and M. Haardt, "Transmit strategies for fullduplex point-to-point systems with residual self-interference," in Proc. International ITG Workshop on Smart Antennas (WSA 2013), pp. 1-8, Mar. 2013.

[8] J. Zhang, O. Taghizadeh, and M. Haardt, "Robust transmit beamforming design for full-duplex point-to-point MIMO systems," Tenth International Symposium on Wireless Communication Systems (ISWCS 2013), pp. 1-5, Aug. 2013.

[9] D. W. Bliss, P. A. Parker, and A. R. Margetts, "Simultaneous transmission and reception for improved wireless network performance," in Proc. IEEE 14th Workshop on Statistical Signal Processing, pp. 478-482, Aug. 2007.

[10] Y. Hua, Y. Ma, P. Liang, and A. C. Cirik, "Breaking the barrier of transmission noise in full-duplex radio," IEEE Military Commun. Conf. (MILCOM), pp. 1558-1563, Nov. 2013.

[11] M. Jain, J. I. Choi, T. Kim, D. Bharadia, K. Srinivasan, S. Seth, P. Levis, S. Katti, and P. Sinha, "Practical, real-time, full duplex wireless," in Proc. Mobicom, pp. 301-312, Sept. 2011.

[12] M. Duarte, C. Dick, and A. Sabharwal, "Experiment-driven characterization of full-duplex wireless systems," IEEE Trans. Wireless Commun., vol. 11, no. 12, pp. 4296-4307, Dec. 2012.

[13] Y. Hua, P. Liang, Y. Ma, A. Cirik, and Q. Gao, "A method for broadband full-duplex MIMO radio," IEEE Signal Process. Lett., vol. 19, no. 12, pp. 793-796, Dec 2012.

[14] D. Bharadia and S. Katti, "Full duplex MIMO radios," USENIX NSDI, pp. 359-372, April, 2014

[15] C.-E. Chen and W.-H. Chung, "An iterative minmax per-stream MSE transceiver design for MIMO interference channel," IEEE Wireless Commun. Lett., vol. 1, no. 3, pp. 229-232, Jun. 2012.

[16] F. Negro, S. P. Shenoy, I. Ghauri, and D. T. M. Slock, "On the MIMO interference channel," in Proc. Information Theory and Applications Workshop (ITA), pp. 1-9, Jan. 2010.

[17] F. Kelly, "Charging and rate control for elastic traffic, Eur. Trans. Telecommun., vol. 8, pp. 33-37, Jan. 1997.

[18] K.-D. Lee and V. C. M. Leung, "Fair allocation of subcarrier and power in an OFDMA wireless mesh network," IEEE J. Select. Areas Commun., vol. 24, no. 11, pp. 2051-2060, Nov. 2006.

[19] V. K. N. Lau, "Proportional fair space-time scheduling for wireless communications," IEEE Trans. Commun., vol. 53, pp. 1353-1360, Aug. 2005.

[20] S. C. Liew and Y. J. Zhang, "Proportional fairness in multi-channel multi-rate wireless networks-Part I: The case of deterministic channels with application to AP association problem in large-scale WLAN," IEEE Trans. Wireless Commun., vol. 7, pp. 3446-3456, Sept. 2008.

[21] D. P. Bertsekas, Nonlinear Programming, 2nd. edition. Belmont, MA: Athena Scientic, 1995.

[22] H. Suzuki, T. V. A. Tran, I. B. Collings, G. Daniels, and M. Hedley, "Transmitter noise effect on the performance of a MIMO-OFDM hardware implementation achieving improved coverage," IEEE J. Sel. Areas Commun., vol. 26, pp. 867-876, Aug. 2008.

[23] W. Namgoong, "Modeling and analysis of nonlinearities and mismatches in AC-coupled direct-conversion receiver," IEEE Trans. Wireless Commun., vol. 4, pp. 163-173, Jan. 2005.
[24] B. Hassibi and B. M. Hochwald, "How much training is needed in multiple-antenna wireless links?" IEEE Trans. Inf. Theory, vol. 49, pp. 951-963, Apr. 2003. 